\title{
Implementation Lessons for Research and Practice
}

\author{
Rosalyn Bertram ${ }^{1} \cdot$ Karen Blase $^{2} \cdot$ Susie Breitenstein ${ }^{3} \cdot$ Nancy Covell $^{4} \cdot$ Dan Edwards ${ }^{5} \cdot$ Thomas Engell $^{6}$. \\ Suzanne E. U. Kerns ${ }^{7} \cdot$ Mitchell Sarkies $^{8} \cdot$ Robert Scherpbier $^{9} \cdot$ Karin Waldherr $^{10} \cdot$ Caitlin Williams $^{11}$
}

Accepted: 29 April 2021 / Published online: 12 May 2021

(C) The Author(s), under exclusive licence to Springer Nature Switzerland AG 2021

\section{Introduction}

Research that examines enactment of policy and practice across disciplines, global regions, sectors, and settings is essential to further advance the science of implementation. We developed Global Implementation Research and Applications (GIRA), the journal of the Global Implementation Society, to identify and expand the diverse ways in which implementation science is understood and applied. As a Springer Nature journal, guidance for submission of research studies to our newly launched journal is available at https://www.springer.com/journal/43477/submission-guide lines.

We broadly view implementation as the act of carrying an intention of change into effect (Peters et al., 2013). Rigorous, well-planned research is the foundation for every science, and studies of implementation increasingly inform policy and practice across diverse sectors and settings (Bertram et al., 2021). However, adaptations are often necessary and may unfold before they can be examined in a research study. Implementation challenges and strategies and subsequent adaptations, adjustments and lessons from these situations are valuable. They can contribute to contextual and intricate understandings of implementation that may spark new ideas for research and collaboration. In this brief editors' note, we

Rosalyn Bertram

bertramr@umkc.edu

1 School of Social Work, University of Missouri-Kansas City, Kansas City, USA

2 Active Implementation Research Network, Chapel Hill, USA

3 Ohio State University, Columbus, USA

4 Columbia University Vagelos College of Physicians and Surgeons and NYSPI, New York, USA

5 Evidence Based Associates, Washington DC, USA

6 Regional Centre for Child and Adolescent Mental Health, Department of Psychology, University of Oslo, Oslo, Norway describe the types of manuscripts that, in addition to planned research studies, are welcome for submission to this journal.

\section{Implementation Adaptations and Adjustments}

Implementation lessons, perspectives, and practices emerge as events unfold, as new strategies and tactics are tried, and as data are collected and analyzed for impact. For example, lessons emerge when teachers identify missing supports for applying a new curriculum and then make practical, effective adjustments as they deliver it. Similarly, after clinical training events, supervisors and practitioners may identify missing implementation supports and then refine case data so it documents key program elements to inform coaching while monitoring proximal outcomes. In another example, response to the COVID-19 pandemic forced rapid adjustments to academic and field instruction in professional degree programs, as well as adaptations made by for-profit and not-for-profit organizations and services across a variety of global regions, sectors and settings. Or consider the seldom documented implementation lessons that continuously unfold amidst changing agendas and interventions that address economic and environmental, political and social concerns.

7 Kempe Center for the Prevention and Treatment of Child Abuse and Neglect, University of Colorado, Aurora, USA

8 Centre for Healthcare Resilience and Implementation Science, Australian Institute of Health Innovation, Faculty of Medicine, Health and Human Sciences, Macquarie University, Sydney, Australia

9 UNICEF, Geneva, Switzerland

10 Ferdinand Porsche FernFH, Distance Learning University of Applied Sciences, Wiener Neustadt, Austria

11 Department of Maternal and Child Health, Gillings School of Global Public Health, University of North Carolina, Chapel Hill, USA 
Each of these examples may appear to be unique, yet each could enhance our knowledge when implementation serves as the common denominator. Well-documented implementation lessons from the field inspire new thinking, inform future implementation efforts, spark future collaborations, and shape necessary rigorous, well-planned research to advance the science and practice of implementation. To expand the perceived boundaries and applications of this science, GIRA encourages potential authors to share the broad scope of their diverse experiences and implementation perspectives.

\section{Manuscripts that Present Implementation Applications}

GIRA invites submissions that document experiences, lessons and perspectives of diverse groups engaged in implementation practice. These may include applications of implementation theory and frameworks to guide policy, programs, projects, or practice, as well as evaluation and use of implementation strategies, pathways, tools, and technologies. Manuscripts also may address contemporary social issues such as the exploration of approaches that challenge systemic racism or the adaptation of implementation strategies to pandemic conditions or in conditions of social unrest.

Manuscripts documenting implementation applications contribute to development of a shared, practical understanding and appreciation of implementation science. They help bridge the frequently noted research-to-practice gap and can create opportunities for collaborative research agendas and partnerships. Documentation of implementation in practice may be authored by consultants, facilitators, or trainers working with program purveyors or policymakers. These manuscripts may also be authored by administrators, consumers, funders, managers, practitioners, or others. By clearly describing the implementation context and change strategies applied, these manuscripts can provoke critical thought and expand our understanding of implementation. In addition, it is important for authors to consider and discuss implications for other disciplines, global regions, sectors, or settings.

\section{Types of Submissions}

Manuscripts should focus on implementation factors that promote and support intended change including adjustments to structures and processes used by organizations and policymakers. They may discuss implementation policy and practice adaptations, applications and syntheses of the literature, and related analysis of proximal outcomes. While we have not established strict page limits, we encourage concise, well-organized manuscripts that are supported by peer-reviewed implementation literature. Submissions that solely focus on an intervention, program, or practice without discussion of implementation factors and literature will not be published.

\section{Case Studies}

These manuscripts examine specific applications of implementation theory, frameworks, tools, or digital technologies to develop, sustain, and improve proximal and distal outcomes. They might pertain to single sites or multi-site applications. They may contrast and compare implementation strategies used across different interventions, settings, or geographic areas. They may offer technical descriptions of local tailoring and adaptation. In all cases, they provide valuable lessons, in-depth understanding of implementation strategies, and provoke new ideas that suggest future implementation research, practice, and policy direction.

\section{Commentaries}

These submissions pose questions and offer perspectives to generate discussion, ideas, and research. Commentaries spark creativity, energize and initiate new ideas and directions.

\section{Policy Notes}

These submissions provide a concise summary of specific implementation issues (e.g., adaptation, infrastructure, scaling) and the policy recommendations to address them. They may inform policymakers directly, as well as associations, foundations, technical assistance entities, intermediaries, practice settings, or others seeking to support or enhance the implementation and sustainment of effective practice. The depth of policy analysis will depend upon settings and the topic of interest. We encourage manuscript submissions that provide unique insights or new perspectives on the interface between policy and implementation.

\section{Research Notes}

These manuscripts may advance a new idea, theoretical perspective, research program, or methodological approach prior to a planned test in a larger study. These brief reports may present preliminary results, null-findings, rapid reviews, or small studies. Research notes function as an incubator or laboratory for new thinking. They may emerge as implementation unfolds and therefore may be more methodologically limited but must offer valuable and thought-provoking contributions to the future study of implementation. 


\section{Potential Topics}

Below is a brief list of potential topics or situations for these types of manuscripts. They illustrate practical implementation topics frequently not submitted for publication because the implementation adaptations occur without the rigorous examination typically associated with funded research studies. These examples were presented in recent discussions with potential authors at the University of South Florida's Implementation and Translational Research Education Conference as GIRA's first issue reached publication, as well as in two editors' panel discussions at the May 2021 Global Implementation Conference. There are, of course, any number of additional possibilities.

- Academic, intermediary, and technical assistance organizational efforts to develop implementation specialists' competencies.

- Cultural adaptations of implementation strategies to increase meaningful engagement with and acceptance by diverse communities.

- Development and use of innovative implementation tools and technologies.

- Implementation of an emergent and/or time-limited response to a significant and unplanned disruption impacting services and systems (e.g., natural disaster, social unrest).

- Implementation strategies used to develop, sustain, and improve effective, equitable, culturally appropriate programs and practices.

- Lessons from specific implementation strategies, including those that focus on scaling and sustainability.

- Reports of implementation in less researched, underrepresented populations or settings (e.g., humanitarian relief efforts, social or environmental justice, resourcechallenged settings).

\section{Broad and Diverse Readership}

All manuscripts should consider GIRA's broad and diverse readership in a variety of disciplines, global regions, sectors, and settings. Before submitting a manuscript, we strongly recommend that it be read for understanding and clarity by persons unfamiliar with the discipline or setting. Often terms and phrasing familiar to authors may not be understood by readers from other nations, regions, sectors, or disciplines. Please keep in mind that GIRA authors and readers include but are not limited to:

- Administrators and managers in organizations that are responsible for developing implementation-informed service system infrastructure that supports, sustains, and improves the use of best evidence in practice.

- Evaluators, purveyors, intermediaries, and researchers engaged in community and consumer partnerships and the development and execution of implementation research agendas.

- Policymakers and funders (e.g., government, foundations, charitable sector) interested in creating hospitable environments to improve, scale, and sustain effective, equitable services.

- Practitioners (e.g., clinicians, educators, supervisors) curious about the conditions and supports that promote confidence and competence in delivering and sustaining effective services.

- Professors and students applying implementation science and frameworks as a foundation for professional workforce development.

- Technical assistance providers using implementation best practices to promote adoption, sustainment, and improvement of effective innovations.

\section{Summary}

We hope this brief discussion of potential manuscripts that present diverse implementation applications sparks the creativity and desire to share your experiences and perspectives. In our first issues, there are also some examples. These include discussions of pandemic adaptations to implementation of Functional Family Therapy (Robbins \& Midouhas, in press) and to assessment of organizational readiness (Kolodny-Goetz et al., in press), as well as adjustments to implementation support by intermediary organizations (Covell et al., 2021), and adaptations made to scale up evidencebased practices (Margolies et al., 2021).

We look forward to reviewing your submissions as together we promote the application of implementation science best practices and innovative strategies across a broad range of disciplines, global regions, sectors, and settings. Queries about potential manuscripts may be sent directly to GIRA's editor-in-chief, Rosalyn Bertram PhD, at bertramr@ umkc.edu.

\section{References}

Bertram, R., Edwards, D., Engell, T., Kerns, S. E., Øvretveit, J., RojasAndrade, R., et al. (2021). Welcome to global implementation research and applications. Global Implementation Research and Applications, 1(1), 1-4.

Covell, N. H., Foster, F., McGovern, M., Lopez, L. O., Shaw, R., \& Dixon, L. B. (2021). Intermediary organizations can support integration of fidelity self-assessment and quality improvement. Global Implementation Research and Applications, 1(1), 30-37. 
Kolodny-Goetz, J., Hamm, D. W., Cook, B. S., \& Wandersman, A. (in press). The readiness, resilience and recovery tool: An emerging approach to enhance readiness amidst disruption. Global Implementation Research and Applications, 1(2).

Margolies, P. J., Covell, N. H., \& Patel, S. R. (2021). Applying implementation drivers to scale-up evidence-based practices in New York state. Global Implementation Research and Applications, l(1), 53-64.
Peters, D. H., Adam, T., Alonge, O., Agyepong, I. A., \& Tran, N. (2013). Implementation research: What it is and how to do it. $B M J, 347$, f6753. https://doi.org/10.1136/bmj.f6753.

Robbins, M. S., \& Midouhas, H. M. (in press). Adapting the delivery of functional family therapy around the world during a global pandemic. Global Implementation Research and Applications, 1(2). 